\title{
Intestinal microbiota and kidney diseases
}

\author{
Myung-Gyu Kim, Jihyun Yang, Sang-Kyung Jo \\ Division of Nephrology, Department of Internal Medicine, Korea University College of Medicine, Seoul, Republic of Korea
}

\begin{abstract}
Large microbial communities reside in the gut as an endogenous organ and interact with the host physiology through symbiotic relationships, affecting health. Recent advances in high-throughput sequencing techniques have made it possible to better understand these complex microbial communities and their effects on hosts. Animal and clinical studies have provided considerable evidence to show that the microbiota plays an important role in chronic kidney disease, acute kidney injury, nephrolithiasis, and kidney transplantation by altering the functions of the intestinal barrier, regulating local and systemic inflammation, controlling production of metabolic components, and affecting immune responses. Although the exact mechanism underlying the microbial shift and its impact on disease progression remains uncertain, the kidney-gut interaction clearly plays a significant role in onset and progression of kidney disease and, therefore, holds promise as a therapeutic target. Here, we review recent literature pertaining to the bidirectional relationship between microbes and humans in various kidney diseases and discuss the future direction of microbial research in nephrology.
\end{abstract}

Keywords: Acute kidney injury, Chronic kidney disease, Microbiota, Nephrolithiasis, Transplantation

\section{Introduction}

Over 100 trillion microorganisms including bacteria, fungi, and protozoa (collectively called the microbiota) form colonies in the human gut and interact in a complex manner with their host, directly or indirectly affecting host health $[1,2]$. Microbial species are transferred first from the vaginal canal at birth, and these microbial communities keep changing in response to various environmental stimuli including diet, stress, and antibiotic use. The intestinal microbiota is characterized by significant diversity; however, the microbial composition remains relatively stable over time and is similar within families as well as in individuals from a region with common dietary habits $[3,4]$.

The structure of the microbiota is balanced functionally by commensal or adversarial relationships with the host. Therefore, changes in intestinal microbial balance, known as dysbiosis, are associated with an unbalanced intestinal microbiota with quantitative and qualitative changes in the composition and metabolic functions. Dysbiosis can contribute to pathogenesis in various diseases, including obesity, cancer, diabetes, inflammatory bowel disease, asthma, and cardiovascular disease [5]. In this review, the emerging roles of the intestinal microbiota in various kidney diseases and the future of microbiome research are discussed.

Received: March 4, 2021; Revised: March 31, 2021; Accepted: April 12, 2021

Editor: Jeonghwan Lee, Seoul National University, Seoul, Republic of Korea

Correspondence: Sang-Kyung Jo

Department of Internal Medicine, Korea University Anam Hospital, Korea University College of Medicine, 73 Goryeodae-ro, Seongbuk-gu, Seoul 02481, Republic of Korea. E-mail: sang-kyung@korea.ac.kr

ORCID: https://orcid.org/0000-0002-0496-0258

Copyright (C) 2021 by The Korean Society of Nephrology

(a) This is an Open Access article distributed under the terms of the Creative Commons Attribution Non-Commercial and No Derivatives License (http:// creativecommons.org/licenses/by-nc-nd/4.0/) which permits unrestricted non-commercial use, distribution of the material without any modifications, and reproduction in any medium, provided the original works properly cited. 


\section{The microbiota as an endogenous organ}

The intestinal microbiota exerts a variety of functions while maintaining a symbiotic relationship with the host. Colonic microbiota ferment non-digestible carbohydrates including dietary fibers, cellulose, and resistant starch to produce short-chain fatty acids (SCFAs; acetate, propionate, butyrate, etc.). SCFAs function as an energy source for colonocytes, strengthen intestinal barrier integrity, and exert potent anti-inflammatory and immunomodulatory functions [6-8]. The microbiota is involved in the synthesis of various vitamins (vitamin B12, thiamine, riboflavin, and vitamin $\mathrm{K}$ ) and the metabolism of amino acids [9].

The intestinal microbial community contributes to the development and maturation of the immune system. Defects in the development of gut-associated lymphoid tissue, Peyer's patches, and mesenteric lymph nodes in germ-free mice indicate the important role of the microbiota in immune system development. Germ-free mice also displayed defective immunoglobulin A production and increased susceptibility to and mortality from certain pathogenic bacteria, suggesting that the microbiota is important in normal physiological immune response [10]. Epithelial cells and immune cells crosstalk with the microbiota and recognize pathogenic microorganisms or their metabolic products and subsequently increase the production of antimicrobial proteins and inflammatory cytokines through activation of the nuclear factor kappa B (NF-kB) pathway. Sustained immune activation by intestinal bacteria acts as a key immune modulator, activating both proinflammatory and counter-regulatory, anti-inflammatory pathways, and the balance among these signals through the induction of various immune cells is important for the maintenance of normal physiology. Homeostasis of immune response signals exchanged between the microbiota and the host is important for development of secondary lymphoid organs [11-14].

Microbial-epithelial interactions also serve to protect and maintain the intestinal barrier by several mechanisms, including preventing pathogens from attaching to intestinal epithelial cells [15], activating anti-inflammatory pathways [16], and regulating mucus properties [17]. The colonization of germ-free mice with a mixture of Lactobacillus strains has been shown to enhance barrier integrity, suggesting that the microbiota is important in maintaining barrier integrity [18]. In addition to metabolic and immune functions, the intes- tinal microbiota has been shown to contribute to development of the complex enteric nervous system [19].

In summary, as an endogenous organ, the gut and microbiota perform multiple physiological roles in the host, including metabolism, maintenance of barrier integrity, development of the immune system, immune modulation, and maturation of the enteric nervous system. This knowledge has led to the idea that dysbiosis contributes to the pathogenesis of not only intestinal diseases, but also of various metabolic diseases, cancer, inflammatory diseases, and cardiovascular diseases, and that strategies targeting the microbiota hold promise in prevention and treatment of these diseases.

\section{Intestinal microbiota and chronic kidney disease}

Several studies have shown the presence of dysbiosis in animal models and humans with chronic kidney disease (CKD) $[20,21]$. In CKD patients, uremia, intestinal edema, prolonged colonic transit time, dietary restriction of fiber, metabolic acidosis, and frequent use of antibiotics can directly or indirectly contribute to dysbiosis and an altered intestinal environment [21-26].

In an early study, Vaziri et al. [20] found significant differences in the abundance of 190 bacterial operational taxonomic units between end-stage kidney disease (ESKD) and healthy controls. They showed that the abundance of saccharolytic microorganisms such as Lactobacillus and Bifidobacteria decreases in ESKD, whereas that of proteolytic microorganisms such as Clostridium and Bacteroides increases. Although urea supplementation was unable to provoke similar dysbiosis in a mouse model of CKD, accumulation of uremic toxins could negatively affect the growth of commensal bacteria and might be responsible for dysbiosis [21]. A recent study on Chinese ESKD patients reported a reduction of butyrate-producing bacteria including Roseburia, Faecalibacterium, Coprococcus, and Prevotella [27]. Wong et al. [28] showed the relative expansion of bacterial families producing urease, indole, and p-cresol forming enzymes, while the bacterial families producing SCFAs were reduced. Given the toxic effects of these metabolites and the beneficial effects of SCFA, dysbiosis in patients with ESKD is likely to play certain roles in the development of systemic inflammation and uremic symptoms.

The intestinal barrier, composed of a single epithelial lining 
and mucus layer, prevents transmigration of luminal contents while permitting selective absorption of nutrients, water, and electrolytes. Dysbiosis in CKD has been shown to be associated with barrier disruption that potentially leads to transmigration of bacteria or their metabolites [29-32]. Studies have shown the increased permeability to exogenous polyethylene glycol or increased circulating endotoxin levels in patients with CKD, suggesting enhanced transmigration through a disrupted barrier $[29,30]$. Urea directly provokes a decrease in transepithelial resistance of cultured enterocytes, and intestinal edema and regional ischemia in CKD could lead to development of leaky gut [31]. At the molecular level, intestinal barrier disruption has been associated with decreased expression of heat shock protein 70 (HSP70) and claudin-1, increased expression of pore-forming claudin-2, and epithelial apoptosis in the colon of a mouse model of CKD [31,32]. In a recent study on CKD, dysbiosis and barrier disruption were found to be linked to an altered mucosal immune response through activation of inflammatory macrophages with production of inflammatory cytokines [32]. This could potentially lead to systemic inflammation and aggravated cardiovascular/renal complications [30,32-34].

Uremic toxins derived from the gut are associated with poor outcomes of CKD. Protein-bound uremic toxins such as p-cresyl sulfate or indoxyl sulfate, produced by fermentation of tyrosine or tryptophan by intestinal bacteria, are excreted by tubular secretion in the kidney, leading to elevated blood levels in patients with CKD. Indoxyl sulfate has been reported to increase transforming growth factor- $\beta$ expression and oxidative stress, promote smooth muscle cell calcification, and cause endothelial cell dysfunction. These protein-bound uremic toxins ultimately lead to increased risk of cardiovascular diseases, mortality, and CKD progression [35-38]. Trimethylamine $N$-oxide (TMAO), another uremic toxin derived from bacterial metabolism of quaternary amines, has been reported to be associated with increased mortality in patients with CKD $[39,40]$.

Based on these findings, therapeutic strategies targeting the microbiota, including prebiotics, an indigestible food ingredient that induces activation of microorganisms; probiotics, living microorganisms; synbiotics, a combination of prebiotics and probiotics; and adsorbents, which adsorb toxic substances, might be useful in the treatment of CKD. AST-120, an insoluble enteric carbon adsorbent that can suppress the accumulation of indoxyl sulfate, has been shown to delay dialysis initiation and to slow the reduction in glomerular filtration rate despite negative results shown in a recent double-blind controlled trial [41-44]. Probiotic supplementation has recently been shown to improve glucose homeostasis with a decrease of markers of inflammation and oxidative stress in diabetic hemodialysis patients [45]. In a randomized, double-blind, placebo-controlled crossover trial on patients with CKD, synbiotics showed beneficial effects on serum p-cresyl sulfate reduction associated with favorable modifications of fecal microbiota [46]. However, clinical research published to date does not provide strong evidence of the efficacy of pre- or probiotics in patients with CKD, possibly owing to the limited number of studies and small sample sizes [47]. More importantly, a lack of understanding of the characteristics of dysbiosis that have causal relationships with CKD is the most significant hurdle in translational research targeting the microbiome.

Interestingly, Lobel et al. [48] recently showed that dietary interventions that induce posttranslational modifications in microbial enzymes can influence CKD progression by suppressing uremic toxin production without altering the microbiome composition. These results suggest that therapeutic strategies targeting bacterial metabolites or metabolic pathways might be more effective than those affecting the microbiota community. Given that a large body of evidence indicates significance of kidney-gut interactions in patients with CKD, a better understanding of the molecular mechanisms underlying kidney-gut crosstalk, further development in microbiome analysis techniques, and improved bioinformatics tools are needed.

\section{Intestinal microbiota and acute kidney injury}

Unlike CKD, few studies have analyzed the kidney-gut crosstalk in acute kidney injury (AKI). In a recent study by Yang et al. [49], intestinal dysbiosis, characterized by an increase in Enterobacteriaceae and a decrease in Lactobacilli and Ruminococcaceae, was induced on day 1 in a mouse ischemia/reperfusion injury (IRI) model. Furthermore, the authors showed that germ-free mice transplanted with feces from IRI mice developed more severe postischemic kidney injury compared to controls. These results show that sudden changes in kidney function or injury are sufficient to provoke dysbiosis in a short time period, and the changes in microbial composition might serve as an important modifier 
of AKI. In support of these findings, the authors also showed that depletion of the microbiota using a combination of nonabsorbable antibiotics before IRI significantly reduced postischemic injury. In the same study, the authors showed that kidney IRI-induced dysbiosis is associated with leaky gut, bacterial translocation, and reduced fecal SCFA levels as well as activation of both innate and adaptive immune responses. Neutrophils and proinflammatory macrophages were shown to accumulate in the lamina propria of the large intestine, and the Th17 pathway was shown to be activated in the small intestine. Microbial depletion led to inhibition of Th17 activation and decreased proinflammatory macrophage accumulation in the intestine; simultaneously, it increased the levels of regulatory T cells and M2 macrophages in both the kidney and large intestines. These data suggest that an altered mucosal immune response associated with dysbiosis is an important player in the kidney-gut crosstalk in AKI [49]. Additionally, the data also indicate a shift in the microbiota and in mucosal immunity toward dysbiosis and proinflammatory changes in IRI-induced AKI, which could further aggravate kidney injury.

The gut-kidney crosstalk in AKI is supported by several recent studies that demonstrated the renoprotective effects of probiotics or gut microbiota-derived metabolites [50,51]. Lactobacillus salivarius BP121 was shown to mitigate cisplatin-induced kidney injury by decreasing kidney inflammation, oxidative stress, and serum levels of uremic toxins [50]. Among gut microbiota-derived metabolites, administration of SCFAs (acetate, propionate, and butyrate) mitigated kidney injury, and the renoprotective effects of these molecules were associated with increased autophagy, decreased inflammation, and decreased oxidative stress [51]. In a study by Nakade et al. [52], gut microbiota-derived D-serine was shown to attenuate tubular damage in AKI. Altogether, these data suggest that the gut microbiota is important and could serve as a therapeutic target in AKI. However, further studies that enhance our understanding of the complex kidney-gut interplay are necessary to apply these findings in the treatment of human AKI.

\section{Intestinal microbiota and nephrolithiasis}

Nephrolithiasis is a relatively common kidney disease reported in $6.0 \%$ of the male population and $1.8 \%$ of the female population in Korea [53]. The incidence of the disease increases approximately three fold in individuals with a family history of the disease [54]. The genetic predisposition or environmental conditions shared by family members can influence disease pathophysiology. The concentrations of urinary calcium, oxalate, phosphate, and uric acid play an important role in stone formation, and emerging evidence indicates active participation of the gut/microbiome in the pathogenesis of nephrolithiasis. Oxalate, which is a constituent of the most common type of kidney stone, is excreted via the urine after absorption in the intestine. Lack of commensal bacteria with oxalate-degrading activity has been shown to be associated with stone formation. In uric acid excretion, one-third of the uric acid is degraded by intestinal uricolysis, also suggesting the possible role of intestinal microbiota in the pathogenesis of uric acid stones. Observations have shown that the overall microbial composition in patients with kidney stones is considerably different from that in healthy controls, which further support the intestinal microbiota as an important contributor to stone formation $[55,56]$. According to a recent systematic review of 25 studies, increase in Enterobacteriaceae and Streptococcaceae and decrease in Prevotellaceae, Prevotella, and Roseburia are characteristic of the microbiota in patients with stone formation [56]. Oxalobacter formigenes are gram-negative, anaerobic bacteria that degrade and, therefore, reduce absorption and subsequent urinary excretion of oxalate, leading to a potential protection against calcium oxalate stone formation $[57,58]$. A case-controlled study in which 47 patients with recurrent calcium oxalate stones were compared with 259 controls showed that colonization with $O$. formigenes reduced the risk of recurrent calcium oxalate stone formation by approximately $70 \%$ [58]. However, even though that preliminary study conducted in a small sample size showed that supplementation with $O$. formigenes significantly reduced urinary or plasma oxalate level, a recent randomized trial on patients with primary hyperoxaluria reported no beneficial effects of $O$. formigenes supplementation $[59,60]$.

Although single microbial strains might not sufficiently lower the pathological risk of oxalate metabolism, therapeutic trials on microbiota modulation strategies, such as treatment with a combination of different microbial strains, diet control, and fecal transplantation, hold promise [61-63], and the intestinal environment can be considered a novel therapeutic target in nephrolithiasis. 


\section{Intestinal microbiota and kidney transplantation}

Dysbiosis in patients with CKD has emerged as an important contributing factor in chronic inflammation and increased cardiovascular risk. However, considering the more complex clinical conditions of transplant recipients, such as improvement of uremia, administration of immunosuppressive drugs, and frequent use of antibiotics, understanding the role of the intestinal microbiota and its interaction with the host immune system or patient outcomes poses a significant challenge [64]. Fricke et al. [65] reported drastic changes in the microbiota in patients in the first month after kidney transplantation (KT) in association with improvement of renal function upon administration of prophylactic antibiotics and high-dose immunosuppressants. However, persistent exposure to immunosuppressants and various posttransplant complications can lead to substantial longitudinal changes in microbial composition [66].

In KT recipients, the balance between activation of allogeneic immune responses and suppression using immunosuppressants is a key factor in determining graft outcomes. Therefore, considering the important immune-modulatory role of intestinal microbiota and various immune cells, it is possible that alterations in the number and composition of microbiota could have a huge impact on graft outcome including transplant rejection and posttransplant infection.

Lee et al. [67] reported that the abundance of Enterococcus in rectal stool samples was associated with urinary tract infection, and the absence of Bacteroides, Ruminococcus, Coprococcus, and Dorea was associated with post-renal transplant diarrhea. In addition to intestinal microbiota, Diaz et al. [68] have demonstrated that long-term administration of immunosuppressants facilitates oral colonization of opportunistic pathogens, leading to increased posttransplant secondary infections.

Other observations in which microbial distance between donor and recipient showed a significant negative correlation with 6-month estimated glomerular filtration rate, suggesting that intestinal microbiota similarity might affect graft outcome [69]. In the same study, the dissimilarity of microbiota between donor and recipient was associated with increased posttransplant infection rate. Moreover, certain microbial species have been shown to affect the blood levels of immunosuppressants by modulating drug pharmacokinetics, which might explain the interindividual differences in tacrolimus dose administered for achieving therapeutic efficacy [70].

The presence of certain species in the microbiota prior to KT has been reported to be significantly associated with subsequent rejection, suggesting a possible role for microbiota in immune modulation [66]. However, despite growing evidence, the effects exerted by the microbial community on immune activation, rejection, or pharmacokinetics remain largely unknown. Future studies assessing the impact of longitudinal changes in individuals and the role of specific microbes in a larger population will help elucidate the role of the microbiota in KT.

\section{Conclusions}

Advances in high-throughput sequencing technology have provided unprecedented insights into the complex microbial communities of the various mucosal surfaces. Similar to several other metabolic and chronic inflammatory conditions including diabetes, obesity, or rheumatoid arthritis, emerging data have demonstrated that alteration of intestinal microbiota is associated with a variety of kidney diseases. Dysbiosis and associated barrier dysfunction, bacterial translocation, and an altered immune response were shown to play important roles in both AKI and CKD. Several strains of bacteria participating in degradation of oxalate have been shown to be associated with oxalate stone formation, and recent studies also suggest the presence of more complex interactions between the microbiota and kidney in transplantation recipients (Fig. 1). However, many of these studies only show a correlation, and causal relationship remains largely unclear. To develop microbiota-targeted therapeutics, further studies unraveling the mechanisms underlying the shifts of microbiota, metabolites, and their impact on disease pathogenesis are needed.

Despite promising data from several clinical trials testing the effect of pre-, pro-, and synbiotics in various kidney diseases, they are derived from studies that enrolled only small numbers of patients; also, the results are inconsistent and limited. Various factors such as individual genetic characteristics, race, and environmental factors complicate the interaction between the microbial community and the host, and targeting a single microbial community might not provide sufficient control over complex host and microbial interactions. 


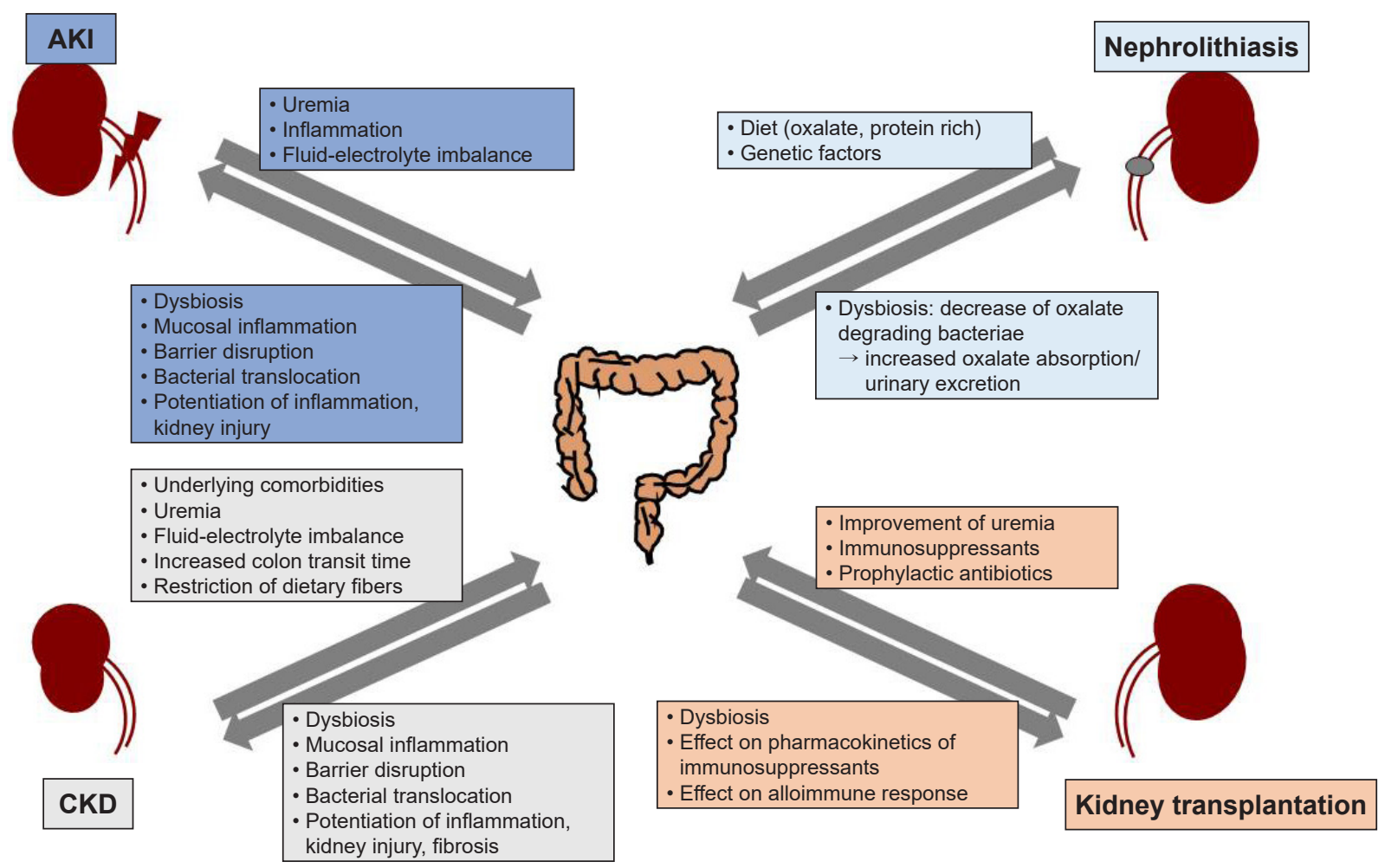

Figure 1. Bidirectional relationships between gut microbiota and various kidney diseases.

AKI, acute kidney injury; CKD, chronic kidney disease.

Nevertheless, the microbiota of mucosal surfaces is a previously unrecognized factor that can potentially modify pathogenesis and outcome in various kidney diseases. A better understanding of the molecular mechanisms linking altered microbiota and its crosstalk with hosts, as well as improved animal models and analytical techniques, should be developed to facilitate translation of these findings to humans.

\section{Conflicts of interest}

All authors have no conflicts of interest to declare.

\section{Funding}

This work was supported by the Basic Science Research Program through the National Research Foundation of Korea funded by the Ministry of Education (2017R1A2B1002734).

\section{Authors' contributions}

Conceptualization: MGK, SKJ

Writing-original draft: MGK, SKJ

Writing-review \& editing: All authors

All authors read and approved the final manuscript.

\section{ORCID}

Myung-Gyu Kim, https://orcid.org/0000-0001-8807-2452

Jihyun Yang, https://orcid.org/0000-0002-8616-238X

Sang-Kyung Jo, https://orcid.org/0000-0002-0496-0258

\section{References}

1. Ley RE, Peterson DA, Gordon JI. Ecological and evolutionary forces shaping microbial diversity in the human intestine. Cell 2006;124:837-848.

2. Mueller NT, Bakacs E, Combellick J, Grigoryan Z, Dominguez-Bello MG. The infant microbiome development: mom 
matters. Trends Mol Med 2015;21:109-117.

3. Human Microbiome Project Consortium. Structure, function and diversity of the healthy human microbiome. Nature 2012;486:207-214.

4. Yatsunenko T, Rey FE, Manary MJ, et al. Human gut microbiome viewed across age and geography. Nature 2012;486:222-227.

5. Durack J, Lynch SV. The gut microbiome: relationships with disease and opportunities for therapy. J Exp Med 2019;216:20-40.

6. den Besten G, van Eunen K, Groen AK, Venema K, Reijngoud DJ, Bakker BM. The role of short-chain fatty acids in the interplay between diet, gut microbiota, and host energy metabolism. J Lipid Res 2013;54:2325-2340.

7. Kim CH, Park J, Kim M. Gut microbiota-derived short-chain Fatty acids, T cells, and inflammation. Immune Netw 2014;14:277288.

8. Morrison DJ, Preston T. Formation of short chain fatty acids by the gut microbiota and their impact on human metabolism. Gut Microbes 2016;7:189-200.

9. Rowland I, Gibson G, Heinken A, et al. Gut microbiota functions: metabolism of nutrients and other food components. Eur J Nutr 2018;57:1-24.

10. Round JL, Mazmanian SK. The gut microbiota shapes intestinal immune responses during health and disease. Nat Rev Immunol 2009;9:313-323.

11. Lotz M, Gütle D, Walther S, Ménard S, Bogdan C, Hornef MW. Postnatal acquisition of endotoxin tolerance in intestinal epithelial cells. J Exp Med 2006;203:973-984.

12. Knauf F, Brewer JR, Flavell RA. Immunity, microbiota and kidney disease. Nat Rev Nephrol 2019;15:263-274.

13. Honda K, Littman DR. The microbiota in adaptive immune homeostasis and disease. Nature 2016;535:75-84.

14. Belkaid Y, Hand TW. Role of the microbiota in immunity and inflammation. Cell 2014;157:121-141.

15. Sherman PM, Johnson-Henry KC, Yeung HP, Ngo PS, Goulet J, Tompkins TA. Probiotics reduce enterohemorrhagic Escherichia coli O157:H7- and enteropathogenic E. coli O127:H6-induced changes in polarized T84 epithelial cell monolayers by reducing bacterial adhesion and cytoskeletal rearrangements. Infect Immun 2005;73:5183-5188.

16. Arnal ME, Lallès JP. Gut epithelial inducible heat-shock proteins and their modulation by diet and the microbiota. Nutr Rev 2016; 74:181-197.

17. Paone P, Cani PD. Mucus barrier, mucins and gut microbiota: the expected slimy partners? Gut 2020;69:2232-2243.

18. Bernet MF, Brassart D, Neeser JR, Servin AL. Lactobacillus aci- dophilus LA 1 binds to cultured human intestinal cell lines and inhibits cell attachment and cell invasion by enterovirulent bacteria. Gut 1994;35:483-489.

19. De Vadder F, Grasset E, Mannerås Holm L, et al. Gut microbiota regulates maturation of the adult enteric nervous system via enteric serotonin networks. Pro Natl Acad Sci USA 2018;115:64586463.

20. Vaziri ND, Wong J, Pahl M, et al. Chronic kidney disease alters intestinal microbial flora. Kidney Int 2103;83:308-315.

21. Chaves LD, McSkimming DI, Bryniarski MA, et al. Chronic kidney disease, uremic milieu, and its effects on gut bacterial microbiota dysbiosis. Am J Physiol Renal Physiol 2018;315:F487F502.

22. Camerotto C, Cupisti A, D’Alessandro C, Muzio F, Gallieni M. Dietary fiber and gut microbiota in renal diets. Nutrients 2019;11:2149.

23. Jernberg C, Löfmark S, Edlund C, Jansson JK. Long-term impacts of antibiotic exposure on the human intestinal microbiota. $\mathrm{Mi}$ crobiology (Reading) 2010;156:3216-3223.

24. Wu MJ, Chang CS, Cheng CH, et al. Colonic transit time in longterm dialysis patients. Am J Kidney Dis 2004;44:322-327.

25. Goraya N, Wesson DE. Dietary management of chronic kidney disease: protein restriction and beyond. Curr Opin Nephrol Hypertens 2012;21:635-640.

26. Gonçalves S, Pecoits-Filho R, Perreto S, et al. Associations between renal function, volume status and endotoxaemia in chronic kidney disease patients. Nephrol Dial Transplant 2006;21:2788-2794.

27. Jiang S, Xie S, Lv D, et al. Alteration of the gut microbiota in Chinese population with chronic kidney disease. Sci Rep 2017;7:2870.

28. Wong J, Piceno YM, DeSantis TZ, Pahl M, Andersen GL, Vaziri ND. Expansion of urease- and uricase-containing, indoleand p-cresol-forming and contraction of short-chain fatty acid-producing intestinal microbiota in ESRD. Am J Nephrol 2014;39:230-237.

29. Magnusson M, Magnusson KE, Sundqvist T, Denneberg T. Impaired intestinal barrier function measured by differently sized polyethylene glycols in patients with chronic renal failure. Gut 1991;32:754-759.

30. McIntyre CW, Harrison LE, Eldehni MT, et al. Circulating endotoxemia: a novel factor in systemic inflammation and cardiovascular disease in chronic kidney disease. Clin J Am Soc Nephrol 2011;6:133-141.

31. Vaziri ND, Yuan J, Norris K. Role of urea in intestinal barrier dys- 
function and disruption of epithelial tight junction in chronic kidney disease. Am J Nephrol 2013;37:1-6.

32. Yang J, Lim SY, Ko YS, et al. Intestinal barrier disruption and dysregulated mucosal immunity contribute to kidney fibrosis in chronic kidney disease. Nephrol Dial Transplant 2019;34:419428.

33. Ramezani A, Raj DS. The gut microbiome, kidney disease, and targeted interventions. J Am Soc Nephrol 2014;25:657-670.

34. Raj DS, Carrero JJ, Shah VO, et al. Soluble CD14 levels, interleukin 6, and mortality among prevalent hemodialysis patients. Am J Kidney Dis 2009;54:1072-1080.

35. Miyazaki T, Ise M, Hirata M, et al. Indoxyl sulfate stimulates renal synthesis of transforming growth factor-beta 1 and progression of renal failure. Kidney Int Suppl 1997;63:S211-S2114.

36. Muteliefu G, Enomoto A, Jiang P, Takahashi M, Niwa T. Indoxyl sulphate induces oxidative stress and the expression of osteoblast-specific proteins in vascular smooth muscle cells. Nephrol Dial Transplant 2009;24:2051-2058.

37. Yu M, Kim YJ, Kang DH. Indoxyl sulfate-induced endothelial dysfunction in patients with chronic kidney disease via an induction of oxidative stress. Clin J Am Soc Nephrol 2011;6:30-39.

38. Hirata J, Hirai K, Asai H, et al. Indoxyl sulfate exacerbates low bone turnover induced by parathyroidectomy in young adult rats. Bone 2015;79:252-258.

39. Tang WH, Wang Z, Kennedy DJ, et al. Gut microbiota-dependent trimethylamine N-oxide (TMAO) pathway contributes to both development of renal insufficiency and mortality risk in chronic kidney disease. Circ Res 2015;116:448-455.

40. Missailidis C, Hällqvist J, Qureshi AR, et al. Serum trimethylamine-N-oxide is strongly related to renal function and predicts outcome in chronic kidney disease. PloS One 2016;11:e0141738.

41. Ueda H, Shibahara N, Takagi S, Inoue T, Katsuoka Y. AST-120, an oral adsorbent, delays the initiation of dialysis in patients with chronic kidney diseases. Ther Apher Dial 2007;11:189-195.

42. Niwa T, Emoto Y, Maeda K, Uehara Y, Yamada N, Shibata M. Oral sorbent suppresses accumulation of albumin-bound indoxyl sulphate in serum of haemodialysis patients. Nephrol Dial Transplant 1991;6:105-109.

43. Akizawa T, Asano Y, Morita S, et al. Effect of a carbonaceous oral adsorbent on the progression of CKD: a multicenter, randomized, controlled trial. Am J Kidney Dis 2009;54:459-467.

44. Schulman G, Berl T, Beck GJ, et al. Randomized placebo-controlled EPPIC trials of AST-120 in CKD. J Am Soc Nephrol 2015; 26:1732-1746.

45. Soleimani A, Zarrati Mojarrad M, Bahmani F, et al. Probiotic sup- plementation in diabetic hemodialysis patients has beneficial metabolic effects. Kidney Int 2017;91:435-442.

46. Rossi M, Johnson DW, Morrison M, et al. Synbiotics easing renal failure by improving gut microbiology (SYNERGY): a randomized trial. Clin J Am Soc Nephrol 2016;11:223-231.

47. Jia L, Jia Q, Yang J, Jia R, Zhang H. Efficacy of probiotics supplementation on chronic kidney disease: a systematic review and meta-analysis. Kidney Blood Press Res 2018;43:1623-1635.

48. Lobel L, Cao YG, Fenn K, Glickman JN, Garrett WS. Diet posttranslationally modifies the mouse gut microbial proteome to modulate renal function. Science 2020;369:1518-1524.

49. Yang J, Kim CJ, Go YS, et al. Intestinal microbiota control acute kidney injury severity by immune modulation. Kidney Int 2020;98:932-946.

50. Lee TH, Park D, Kim YJ, et al. Lactobacillus salivarius BP121 prevents cisplatin-induced acute kidney injury by inhibition of uremic toxins such as indoxyl sulfate and p-cresol sulfate via alleviating dysbiosis. Int J Mol Med 2020;45:1130-1140.

51. Andrade-Oliveira V, Amano MT, Correa-Costa M, et al. Gut bacteria products prevent AKI induced by ischemia-reperfusion. J Am Soc Nephrol 2015;26:1877-1888.

52. Nakade Y, Iwata Y, Furuichi K, et al. Gut microbiota-derived D-serine protects against acute kidney injury. JCI Insight 2018; 3:e97957.

53. Kim H, Jo MK, Kwak C, et al. Prevalence and epidemiologic characteristics of urolithiasis in Seoul, Korea. Urology 2002;59:517521.

54. Curhan GC. Epidemiology of stone disease. Urol Clin North Am 2007;34:287-293.

55. Stern JM, Moazami S, Qiu Y, et al. Evidence for a distinct gut microbiome in kidney stone formers compared to non-stone formers. Urolithiasis 2016;44:399-407.

56. Stanford J, Charlton K, Stefoska-Needham A, Ibrahim R, Lambert $\mathrm{K}$. The gut microbiota profile of adults with kidney disease and kidney stones: a systematic review of the literature. $B M C$ Nephrol 2020;21:215.

57. Allison MJ, Dawson KA, Mayberry WR, Foss JG. Oxalobacter formigenes gen. nov., sp. nov.: oxalate-degrading anaerobes that inhabit the gastrointestinal tract. Arch Microbiol 1985;141:1-7.

58. Kaufman DW, Kelly JP, Curhan GC, et al. Oxalobacter formigenes may reduce the risk of calcium oxalate kidney stones. J Am Soc Nephrol 2008;19:1197-203.

59. Hoppe B, Beck B, Gatter N, et al. Oxalobacter formigenes: a potential tool for the treatment of primary hyperoxaluria type 1. Kidney Int 2006;70:1305-1311. 
60. Milliner D, Hoppe B, Groothoff J. A randomised Phase II/III study to evaluate the efficacy and safety of orally administered Oxalobacter formigenes to treat primary hyperoxaluria. Urolithiasis 2018;46:313-323.

61. Peck AB, Canales BK, Nguyen CQ. Oxalate-degrading microorganisms or oxalate-degrading enzymes: which is the future therapy for enzymatic dissolution of calcium-oxalate uroliths in recurrent stone disease? Urolithiasis 2016;44:45-50.

62. Stern JM, Urban-Maldonado M, Usyk M, et al. Fecal transplant modifies urine chemistry risk factors for urinary stone disease. Physiol Rep 2019;7:e14012.

63. Lieske JC, Tremaine WJ, De Simone C, et al. Diet, but not oral probiotics, effectively reduces urinary oxalate excretion and calcium oxalate supersaturation. Kidney Int 2010;78:1178-1185.

64. Campbell PM, Humphreys GJ, Summers AM, et al. Does the microbiome affect the outcome of renal transplantation? Front Cell Infect Microbiol 2020;10:558644.

65. Fricke WF, Maddox C, Song Y, Bromberg JS. Human microbiota characterization in the course of renal transplantation. Am J
Transplant 2014;14:416-427.

66. Swarte JC, Douwes RM, Hu S, et al. Characteristics and dysbiosis of the gut microbiome in renal transplant recipients. J Clin Med 2020;9:386.

67. Lee JR, Muthukumar T, Dadhania D, et al. Gut microbial community structure and complications after kidney transplantation: a pilot study. Transplantation 2014;98:697-705.

68. Diaz PI, Hong BY, Frias-Lopez J, et al. Transplantation-associated long-term immunosuppression promotes oral colonization by potentially opportunistic pathogens without impacting other members of the salivary bacteriome. Clin Vaccine Immunol 2013;20:920-930.

69. Kim JE, Kim HE, Cho H, et al. Effect of the similarity of gut microbiota composition between donor and recipient on graft function after living donor kidney transplantation. Sci Rep 2020;10:18881.

70. Lee JR, Muthukumar T, Dadhania D, et al. Gut microbiota and tacrolimus dosing in kidney transplantation. PloS One 2015;10:e0122399. 